\title{
Meta
}

Journal des traducteurs

Translators' Journal

\section{CURRAn, Beverley, SATO-Rossberg, Nana et TANABE, Kikuko, dir. (2015) : Multiple Translation Communities in Contemporary Japan. New York/Londres : Routledge, 224 p.}

\section{Anna Joan Casademont}

Volume 61, numéro 3, décembre 2016

URI : https://id.erudit.org/iderudit/1039227ar

DOI : https://doi.org/10.7202/1039227ar

Aller au sommaire du numéro

Éditeur(s)

Les Presses de l’Université de Montréal

ISSN

0026-0452 (imprimé)

1492-1421 (numérique)

Découvrir la revue

Citer ce compte rendu

Casademont, A. J. (2016). Compte rendu de [CURRAN, Beverley,

SATO-Rossberg, Nana et TANABE, Kikuko, dir. (2015) : Multiple Translation Communities in Contemporary Japan. New York/Londres : Routledge, 224 p.]

Meta, 61(3), 727-728. https://doi.org/10.7202/1039227ar d'utilisation que vous pouvez consulter en ligne. 


\section{DOCUMENTATION}

\section{Comptes rendus}

Curran, Beverley, Sato-Rossberg, Nana et TANABE, Kikuko, dir. (2015): Multiple Translation Communities in Contemporary Japan. New York/Londres: Routledge, $224 \mathrm{p}$.

Multiple Translation Communities in Contemporary Japan est un ouvrage collectif dirigé par Beverley Curran, du Département de société, culture et média de l'Université chrétienne internationale de Tokyo, Nana Sato-Rossberg, de l'École d'études orientales et africaines de l'Université de Londres et Kikuko Tanabe du Collège de Kobe. Ce livre figure dans la collection Advances in Translation Studies de Routledge qui promeut des recherches sur différentes formes de traduction et leurs enjeux éthiques, idéologiques, politiques, sociologiques, culturels, etc.

Multiple Translation Communities in Contemporary Japan est un recueil d'articles qui nous offre un aperçu varié de différentes pratiques de traduction qui ont lieu entre plusieurs des communautés qui font partie de la société japonaise ou entre ces communautés et d'autres sociétés hors Japon. Les textes présentés traitent d'aspects comme la traduction de différents genres (littérature, manga, cinéma, théâtre, télévision), l'influence des médias, l'usage identitaire et revendicatif des traductions (féminisme, queer, appropriation identitaire), ainsi que la transmission des productions culturelles traditionnelles et populaires japonaises dans la société japonaise contemporaine et ailleurs dans le monde.

Le livre comporte une introduction, dix articles rédigés par différents auteurs suivis d'une brève biographie de chacun ainsi que d'un index thématique.

L'introduction, écrite par Beverly Curran, explique clairement le point de vue des éditrices en ce qui concerne le concept de "traduction", ce qui permet d'argumenter le choix des articles: «Translation is now a mode of engagement animated by multiple texts in a mix of media accessed and $\mathrm{read} /$ viewed/played in multiple orders by multiple communities of readers/viewers/players. Translation is not relegated to the afterlife of a linguistic event; rather, it is now located among a number of cultural practices that "prolong worlds" in the networked present» (p. viii). Les idées de dynamisme, de mouvement et de carrefour (entre disciplines, entre médias, entre cultures, entre communautés, etc.) sont donc très présentes dans les réflexions présentées par les différents auteurs des articles. Cette image du carrefour explique précisément pourquoi, même si les éditrices avaient envisagé de diviser les dix chapitres du livre en trois grandes sections (manga, cinéma et théâtre; genres, communautés queer et traductions croisées; traduction littéraire), cette option n’a pas été retenue, puisque chaque article proposé combine de façon imbriquée plusieurs des axes thématiques mentionnés.

Le premier article, intitulé «Death Note: Multilingual Manga and Multidimensional Translation» de Berverley Curran, porte sur les interactions entre le japonais et l'anglais dans un Japon multilingue et sur ce que signifie l'idée de langues nationales dans le contexte actuel d'échanges transnationaux continus. Pour cela, elle analyse la version originale japonaise du manga Death Note et sa traduction en anglais, ce qui lui permet de considérer l'usage des deux langues dans des situations différentes au long de l'histoire du manga en question, ainsi que d'observer le rapport que les personnages eux-mêmes ont avec le multilinguisme présent dans leur quotidien et comment ce rapport est reproduit ou présenté dans la version traduite précisément à l'anglais.

Le deuxième texte, «Litterature and Theatre into Film: Shindô Kaneto's Kuroneko » de Titanilla Mátrai, permet d'explorer la traduction entre différents genres et la traduction d'éléments propres à la culture traditionnelle et populaire japonaise à des époques plus actuelles. Spécifiquement, la recherche examine le film Yabu no naka no kuroneko (1968) de Shindô Kaneto et la façon dont le réalisateur combine des composantes propres au théâtre nô, au kabuki et à des légendes japonaises, avec le sous-genre de films pop d'horreur mettant en vedette des chats monstrueux très en vogue dans les années 1960.

Toujours dans le contexte de la traduction entre genres, le troisième article, intitulé «Translating Kamui Gaiden: Intergeneric Translation from Manga to Live-Action Film» de Nana SatoRossberg), porte sur la reconstruction du concept du héros à partir du personnage principal du manga Kamui Gaiden des années 1960, créé par Shirato Sanpei, en le comparant avec l'adaptation filmique qui en a été faite 50 ans plus tard. L'exemple choisi permet d'observer comment les changements culturels entre les différentes générations réceptrices et du manga et du film jouent un 
rôle important dans la façon dont la transmission de l'idée du héros est émise et reçue.

Quant aux théories féministes et la traduction, les articles «The Revolution Cannot Be Translated: Transfiguring Discourses of Women's Liberation in 1970-1980s Japan» écrit par James Welker et «Catherine MacKinnon in Japanese: Toward a Radical Feminist Theory of Translation» de Caroline Norma explorent la traduction en tant qu'activité nécessitant dialogue et interaction (ix). Les deux études considèrent les traductions de textes féministes à différents moments de l'histoire du Japon et à quel point ces traductions (choix des textes traduits, présentations d'idées, adaptation de la terminologie) ont été des enjeux importants dans l'appropriation qui en a été faite par les mouvements féministes nippons.

En ce qui concerne les mouvements queer, le sixième texte, "Translating Queer in Japan: Affective Identification and Translation in the 'Gay Boom' of the 1990s» de Jeffrey Angles, aborde les liens de communauté et de solidarité tissés (dans le cas de son article dans les années 1980 et 1990) entre les lecteurs de textes du mouvement, en japonais et en anglais, ainsi qu'entre les membres de la communauté de traducteurs qui se chargeaient des textes en question et qui permettaient, donc, leur diffusion dans les deux langues.

Dans le même contexte queer, Claire Maree, dans «The Perils of Paisley and Wierd Manwomen: Queer Crossings into Primetime J-TV via Telops », analyse l'évolution de l'onê-kotoba (un style de discours japonais propre à la communauté queer) vers l'usage grand public qui s'en fait dans les émissions de télévision et concrètement dans les textes ou graphiques superposés aux images, très utilisés dans les programmes de variétés japonais.

Et dans le domaine des variations linguistiques, qui sont si présentes et fortes au Japon (voir l'article mentionné ci-dessus sur l'onê-kotoba), «Translating Gendered Voices: From Tanizaki Junichirô's Naomi to Yoshimoto Banana's Kitchen" examine l'onna-kotoba (ou le langage des femmes japonaises) dans sa traduction en anglais. Concrètement, Maree analyse les ouvrages Chijin no ai (1925) de Tanizaki Junichiro et Kitchen (1983) de Yoshimoto Banana, et leurs traductions respectives de 1985 et 1993, afin d'observer comment les voix féminines sont gérées dans les versions en anglais.

"Hirai Teiichi, the Japanese Translator of Dracula and Literary Shape-shifter», de Masaya Shimokusu étudie les différentes traductions réalisées par Hirai Teiichi de l'œuvre de Bram Stoker. L'étude contrastive des différentes versions permet de suivre l'intégration du personnage de Dracula dans l'imagerie populaire japonaise, l'évolution de sa perception dans la société nippone et com- ment le traducteur décide de le présenter afin de proposer une traduction attrayante pour le public de chaque époque. Elle permet également d'envisager les conséquences des interactions inévitables entre les différentes versions du livre et d'autres expressions et diffusions de l'œuvre au cinéma au fil des années.

Finalement, Yin-Ji Piao propose «Yun Dongju's poetry in Japanese Translation». Ce dernier article du recueil offre une analyse littéraire comparative entre les poèmes Hangul originaux de Yun Dong-ju en coréen et leur traduction en japonais. Yin-Ji Piao utilise lesdits poèmes comme des exemples lui permettant de généraliser certains enjeux essentiels du point de vue de la traduction entre le coréen et le japonais (linguistiques, historiques, politiques, sociologiques).

Multiple Translation Communities in Contemporary Japan collige donc plusieurs travaux interdisciplinaires qui ont comme point commun une vision englobante, non statique de la traduction, qui est étroitement liée aux constantes interactions entre communautés, qu'elles soient au Japon ou ailleurs.

ANNA JoAn CASAdEMONT Télé-université du Québec, Québec, Canada

Lafarga, Francisco y Pegenaute Luis, eds. (2013): Diccionario histórico de la traducción en Hispanoamérica. Madrid/Fráncfort: Iberoamericana/Vervuert, 515 p.

Como ya hicieran con el Diccionario histórico de la traducción en España (Editorial Gredos) en 2009, Francisco Lafarga y Luis Pegenaute dan a conocer con esta nueva obra la historia de la traducción en Hispanoamérica, y lo hacen mediante la estructuración, reelaboración y ampliación de la información original previa disponible. Dicha información, menos voluminosa y más dispersa que otras en lo que respecta al campo de la Historia de la traducción (en concreto, de la traducción española), proviene de todos los trabajos e investigaciones realizados hasta el momento, no solo en España, sino también en Hispanoamérica. Por lo tanto, de este diccionario enciclopédico destaca la excelente labor de coordinación de autores y de recopilación de datos que han llevado a cabo sus dos editores.

El volumen consta de 214 entradas que se centran, principalmente, en la traducción literaria, aunque también se presta atención a otras obras que, aunque no son puramente literarias, sí entran dentro del ámbito de las humanidades.

Según indican los editores en la introducción (p. 9-10), las entradas se han dividido en los siguientes grupos: 